\title{
Characterization of Geometric Uncertainties of a Dose Calibrator During Measurement of ${ }^{90}$ Y Activity
}

\author{
João A.M. Santos ${ }^{1}$, Anabela G. Dias ${ }^{1}$, and Artur L. Bastos ${ }^{2}$ \\ ${ }^{I}$ Serviço de Física Médica and Centro de Investigação, Instituto Português de Oncologia do Porto Francisco Gentil, E.P.E, Porto, \\ Portugal; and ${ }^{2}$ Serviço de Medicina Nuclear, Instituto Português de Oncologia do Porto Francisco Gentil, E.P.E, Porto, Portugal
}

\begin{abstract}
The performance and measurement of ${ }^{90} \mathrm{Y}$ with a dose calibrator has always been a key point of several international recommendations, specifically in the case of the radiopharmaceutical $90 Y$-ibritumomab tiuxetan, among other applications, used in the treatment of non-Hodgkin lymphoma. The objective of the present work was to examine in detail some important sources of geometric errors that, if neglected, can lead to an increase in uncertainty about the measured activity of this $\beta^{-}$emitter radionuclide. Methods: A CRC-15R dose calibrator was used to measure and quantify some of these sources, although the same methodology can easily be applied to any other similar equipment. The depth response along the main axis of the dose calibrator was carefully characterized, as well as syringe volume effects and source angular dependence. Results: It was found that, if not taken properly into account, these issues can contribute to an increase in the activity uncertainty (e.g., 5.1\% in the present example). This finding implies the possibility of easily reaching higher values than the internationally suggested $5 \%$ uncertainty in activity measurement for therapeutic purposes. Conclusion: These errors can be greatly reduced by previous characterization of the dose calibrator and careful implementation of the methodology for measurement.
\end{abstract}

Key Words: dose calibrator; error propagation; ${ }^{90} \mathrm{Y}$

J Nucl Med Technol 2011; 39:125-130

DOI: $10.2967 /$ jnmt.110.077404

\section{A}

common procedure in nuclear medicine is the use of ${ }^{90} \mathrm{Y}$ to label ibritumomab tiuxetan (Zevalin; Spectrum Pharmaceuticals, Inc.). The radiopharmaceutical ${ }^{90}$ Y-ibritumomab tiuxetan is a stable agent used in the treatment of non-Hodgkin lymphoma. It consists of a murine $\mathrm{IgG}_{1} \kappa$-monoclonal antibody directed to the CD20 antigen that exists in the surface of normal and malignant B cells. ${ }^{90} \mathrm{Y}$ (with a half-life of $64.1 \mathrm{~h}$,

\footnotetext{
Received Mar. 18, 2010; revision accepted Nov. 17, 2010.

For correspondence or reprints contact: João A.M. Santos, Instituto Português de Oncologia do Porto Francisco Gentil, E.P.E, Serviço de Medicina Nuclear, Rua Dr. António Bernardino de Almeida, 4200-072 Porto, Portugal.

E-mail: j.a.miranda.santos@gmail.com

COPYRIGHT @ 2011 by the Society of Nuclear Medicine, Inc.
}

maximum energy of $2.28 \mathrm{MeV}$, and average penetration range of approximately $5 \mathrm{~mm}$ in soft tissue; $90 \%$ of the $\beta^{-}$energy is absorbed in a sphere of $5.3-\mathrm{mm}$ radius) is a quasipure $\beta^{-}$emitter with an internal production branching ratio responsible for 32 annihilations of a $\beta^{+} \beta^{-}$pair in every 1 million decays (1). Recently, the possibility of acquiring ${ }^{90} \mathrm{Y}$ PET images using the $511-\mathrm{keV}$ photons resulting from this process to assess biodistribution of ${ }^{90} \mathrm{Y}$ radiopharmaceuticals after therapy administration was demonstrated $(2,3)$. This possibility increases interest in dose distribution, which in turn increases the importance of an accurate determination of activity. However, treatment with ${ }^{90}$ Y-ibritumomab is administered only after biodistribution confirmation by ${ }^{111}$ In-ibritumomab, and some type of dosimetry estimation, which can be achieved by imaging ${ }^{111} \mathrm{In}$ with conventional nuclear medicine techniques (e.g., Anger camera, either in planar or SPECT mode).

According to several international recommendations (49 ), activity should be determined carefully and the error in the administered activity must not exceed $5 \%$ for therapeutic purposes (10). Because ${ }^{90} \mathrm{Y}$ is nearly a pure $\beta^{-}$emitter, the geometric conditions under which the activity is measured in the dose calibrator are of extreme importance. Because of the clinical relevance, the methodology of measuring ${ }^{90} \mathrm{Y}$ activity, or other radionuclides having a high abundance of $\beta^{-}$radiation, has been addressed often in the literature $(11,12)$. However, its measurement methodology is more demanding for ${ }^{90} \mathrm{Y}$ than for pure $\gamma$-emitters. Also, an effort to more accurately calculate the effective dose to the patient and dose to target organs is also being continuously pursued (13).

The calibration certificate supplied with the radionuclide for labeling the monoclonal antibody is used to calculate the calibration factor for all the vials and syringes used during the labeling procedure as follows. First, the source activity is calculated for the dose calibration time. Then, the activity by unit volume is calculated from the calibration certificate. The vial is then introduced in the dose calibrator and a new calibration factor is determined for that vial. Once the calibration for that particular vial is determined, a certain volume is taken out of the vial with a syringe used 
for the labeling process and its activity is measured. Knowledge of the exact volume extracted from the vial enables determination of the activity inside the syringe. With this information, another calibration factor, corresponding to that particular syringe, is established. This process is repeated for the reaction vial and for the syringe used for dose administration. Several calibration factors are thus obtained for all the containers (syringes and vials) used in the labeling process.

This entire procedure reflects the importance of the method of determining calibration factors, always having in mind a maximum error of $5 \%$ used for activity of ${ }^{90} \mathrm{Y}$ measurement.

The problem with determining a different calibration factor for each container used to measure the activity of a pure $\beta^{-}$source arises from the fact that the measured activity depends mainly on bremsstrahlung radiation. The corresponding photon distribution is critically dependent on the geometry of the source (radius in the case of cylindric sources).

However, even if all the described steps and volume determinations are strictly controlled, several errors might still be present and will affect the activity measurement and thus the dose received by the patient. In fact, the geometry of holding devices inside the dose calibrator can also give rise to nonnegligible or even large sources of errors. This work aimed to characterize some of these uncertainties and error sources not mentioned in the literature for this type of measurement device.

\section{MATERIALS AND METHODS}

A CRC-15R dose calibrator (Capintec; Fig. 1) was used for the experiments. This calibrator consists of a well-type ionizing chamber, a protecting metallic cylinder, an interior plastic insert, and a plastic source holder (vials and syringes). Because of the general nature of these devices, the kinds of geometric errors found in this model will probably be similar to those found in other models.

\section{Depth Profile from Point Source}

Initially, several depth profiles along the axis of the dose calibrator were measured using a point source of ${ }^{90} \mathrm{Y}$ and another of ${ }^{99 \mathrm{~m}} \mathrm{Tc}\left(<1 \mathrm{~mm}^{3}\right)$, according to a method described elsewhere (14). The point sources were encapsulated inside a small acrylic homemade device (1-mm wall thickness) and inserted inside the dose calibrator well. Because volume could not be measured, the activity inside sources could not be measured accurately or the calibration factor determined. However, the activity was about $5 \mathrm{MBq}$, and none of the curve profiles changed with activity variation along several profile measurements with different activities. Also there is the possibility of a nonlinear behavior for higher activities. Unfortunately that possibility could not be verified, because the small size of the point source made a higher activity impossible to obtain. However, this measurement was performed several times, with different

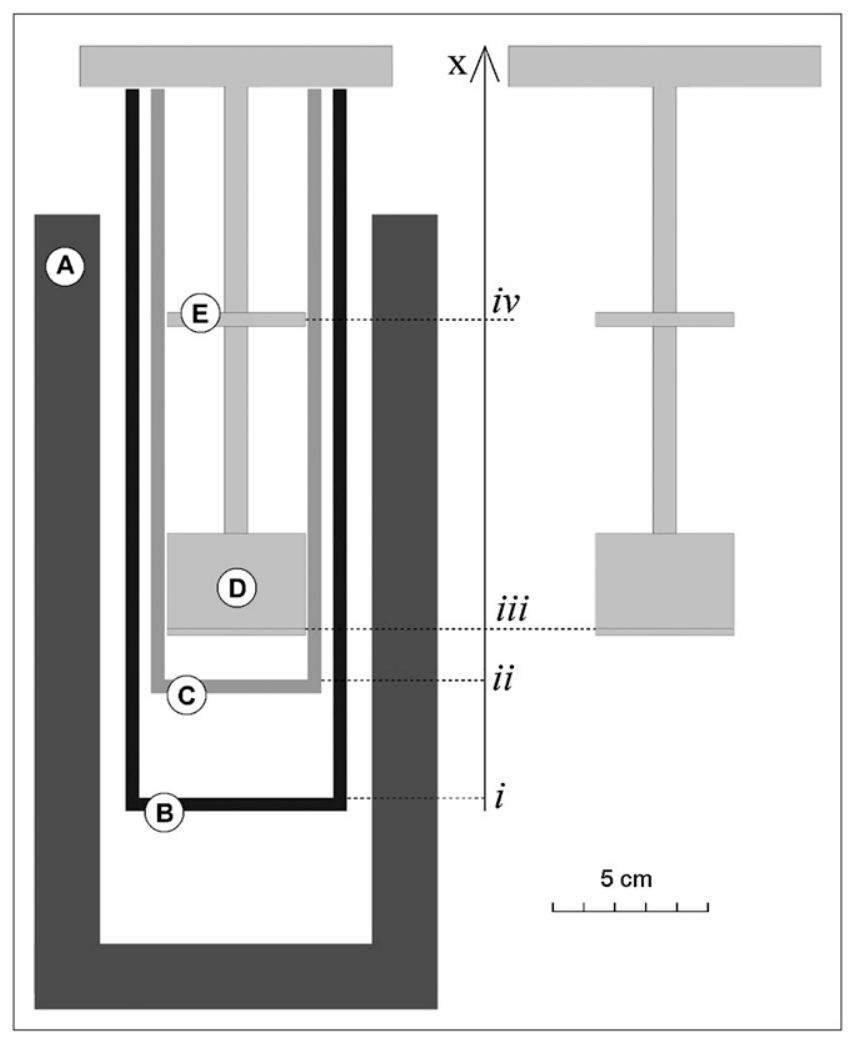

FIGURE 1. Schematic of CRC-15R dose calibrator used in this study. A and B represent ionization chamber and inner metallic wall housings; C represents removable plastic protection for contamination prevention; $D$ and $E$ represent 2 key features of plastic source holder: vial holder and syringe holder, respectively.

activities, depending on the activity volume concentration available, yielding always the same results. The point source position was determined by externally measuring the displacement of the supporting cable from point to point. The depth profiles should be proportional to the depth responsiveness of the detector. The profile normalized by its maximum (or in-depth responsiveness of the detector), $r(x)$, was depicted as a function of the longitudinal distance $x$ from the bottom of the metallic container of the dose calibrator well (shown as B in Fig. 1). With the source positioned as close as possible to the bottom of the metallic surface B, the first measurement point is $r(0)$ (point $i$ in the coordinate axis $x$ in Fig. 1).

Next to the metallic surface of the well, the dose calibrator has a removable plastic insert (shown as $\mathrm{C}$ in Fig. 1) that prevents undesired contamination of nonmovable parts (e.g., metallic surface B) and helps to define the useful range of the measurement (ideally, a constant responsiveness length) inside the dose calibrator well. The ${ }^{90} \mathrm{Y}$ measurements were performed using an arbitrary calibration constant (e.g., ${ }^{131} \mathrm{I}$ ) because the normalized curves are not affected by this parameter. A time decay correction for the measurement period was performed for all the measured apparent activity $\left(A_{\text {app }}\right)$ values. 


\section{Angular Dependence}

One feature observed during the measurement of ${ }^{90} \mathrm{Y}$ sources was the $A_{a p p}$ angular dependence on the source holder position relative to the dose calibrator well. To investigate the magnitude of this effect, we positioned an arbitrary activity of ${ }^{90} \mathrm{Y}$ inside a $10-\mathrm{mL}$ syringe in the center of the source holder and rotated the entire set (source and holder) $360^{\circ}$ (by $10^{\circ}$ steps). An external circular scale was used, and rotation of the holder and source was relative to the fixed dose calibrator well and plastic inset. In addition to the causes of measurement uncertainty already described, an angular dependence on the source holder position was also characterized. The values were taken after a period of stabilization with the source in place at a given angle. After this period, the maximum and minimum values of $A_{a p p}$ fluctuation in the dose calibrator display were recorded.

\section{Comparison of Activity Measurement with 10-mL Syringes}

Because of the variation of the dose calibrator responsiveness $r(x)$ along its longitudinal axis, the $A_{a p p}$ inside the syringes placed at the syringe location on the plastic holder shown in Figure 1 was measured using a methodology previously described in more detail (15): the volume of a syringe (increasing dilution by adding water) with a fixed activity and position was varied from near zero to its maximum.

\section{Total-Error Propagation Estimation}

The final measurement of the actual activity $A$ (directly dependent on choosing the correct calibration factor) will have a total error $\delta A, A_{a p p}=A \pm \delta A$. Taking into account all sources of error and assuming them to be independent ( $A_{\text {app }}=A \pm \delta A_{1}, A_{\text {app }}=A \pm \delta A_{2}$, etc.), and if we calculate each individually ( $\delta A_{1}, \delta A_{2}$, etc.), we can add these uncertainties in quadrature to obtain an overall uncertainty $\delta A$ and write (14):

$$
\frac{\delta A}{A}=\sqrt{\left(\frac{\delta A_{1}}{A}\right)^{2}+\left(\frac{\delta A_{2}}{A}\right)^{2}+\ldots}
$$

\section{RESULTS}

\section{Longitudinal Profile $A_{a p p}$ Dependence with Source Holder}

An initial measurement of ${ }^{90} \mathrm{Y}$ was performed with and without the plastic insert. Three longitudinal in-depth profiles were acquired using point sources of ${ }^{90} \mathrm{Y}$, with and without the plastic insert. The ${ }^{99 \mathrm{~m}} \mathrm{Tc}$ profile with the insert inside the well was acquired for comparison purposes. All the normalized curves can be compared in Figure 2. Because the profiles are normalized to the maximum of each curve, the calibration factor is irrelevant and the same calibration constant $\left({ }^{131} \mathrm{I}\right)$ was used for all the measurements presented in the figure.

Clinical activity measurements of ${ }^{90} \mathrm{Y}$ are always made with the plastic insert (part $\mathrm{C}$ in Fig. 1) and the plastic sample holder (parts D and $\mathrm{E}$ in the same figure). Therefore,

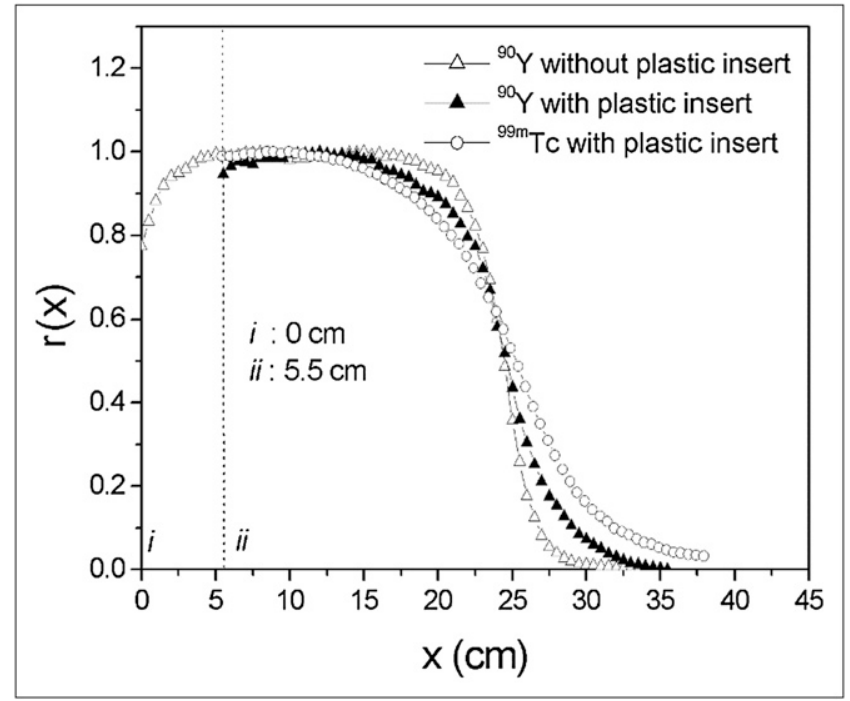

FIGURE 2. Responsiveness curves, $r(x)$, of dose calibrator as function of axial longitudinal distance, $x$. Responsiveness was defined as $A_{a p p}$ normalized by its maximum value. Three different responses of dose calibrator for ${ }^{90} Y$ with and without plastic insert and ${ }^{99 m T c}$ with plastic insert are shown. Points $i$ and ii refer to regions shown in Figure 1.

using the methodology already described, we measured 2 longitudinal (depth) profiles separately with these structures placed inside the well and using the same point source.

The measurements, with and without the sample holder, are shown in Figure 3. The effects of the same sample holder can clearly be seen. We can observe that, above point iii, the curves are similar except at the first points (immediately above iii) corresponding to the effect of the plastic base of the sample holder, where the vials usually are placed for measurement. Also there is a clear dissimilarity around point $i v$ (around $x=20.2 \mathrm{~cm}$ ), corresponding to the $\beta^{-}$and bremsstrahlung $x$-ray attenuation due to the syringe support parts.

The graphic inset in Figure 3 shows, in more detail, the in-depth responsiveness, $r(x)$, for $x>6.9 \mathrm{~cm}$, due to the attenuation by the plastic sample holder bottom, as a percentage of the maximum value of $A_{a p p}(x)$ near $x=14 \mathrm{~cm}$. One notices a variation higher than $25 \%$ between the value at the maximum responsiveness point well outside this plastic container and the bottom of the sample holder.

\section{Comparison of Activity Measurement with 10-mL Syringes}

In Figure 4, one can observe the percentage variation of a 10 -mL syringe $A_{a p p}$ as the volume changes from $0 \mathrm{~mL}$ (scale measurement) to almost its maximum, both for ${ }^{99 \mathrm{~m}} \mathrm{Tc}$ and for ${ }^{90} \mathrm{Y}$. Although one notices a decrease in the $A_{\text {app }}$ in both radionuclides, this decrease is more pronounced in the ${ }^{90} \mathrm{Y}$ case, reaching $7 \%$, whereas for ${ }^{99} \mathrm{~m} \mathrm{Tc}$ only a $4 \%$ change is noticed. This difference is due mostly to the initial behavior of the ${ }^{90} \mathrm{Y}$ curve, for geometric reasons that will be covered in the "Discussion" section. If the calibration factor is obtained with half the syringe full, we 


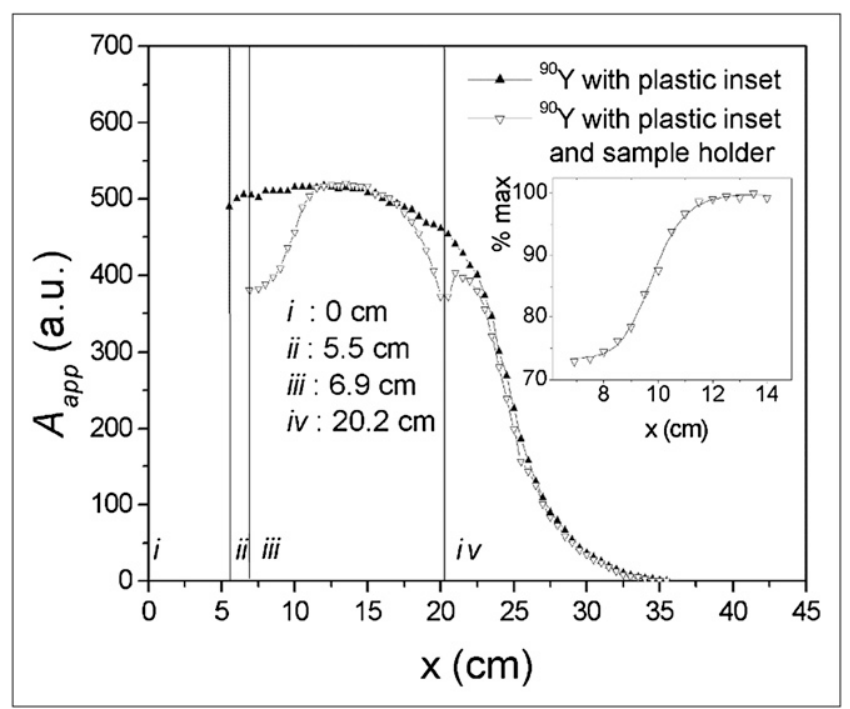

FIGURE 3. $A_{a p p}$ as function of axial longitudinal distance obtained with ${ }^{90} \mathrm{Y}$ point source with and without plastic sample holder. Both cases were obtained using plastic insert. Note difference between the 2 curves. Regions (points) i, ii, iii, and iv (Fig. 1) are also indicated. Inset graphic shows percentage variation relative to maximum value (near $x=14 \mathrm{~cm}$ ) at initial part of curve obtained with plastic holder.

can estimate an error afterward. If the volume changes and the activity is determined with the same calibration factor, an error of roughly $\delta A_{1} / A=0.02(2 \%)$ can be reached, assuming that the measured volumes will not be situated at the limits of the possible syringe positions.

\section{Angular Dependence}

Figure 5 shows the mean value of $A_{a p p}$ as a function of the angle of the source holder. The error bars indicate \pm the

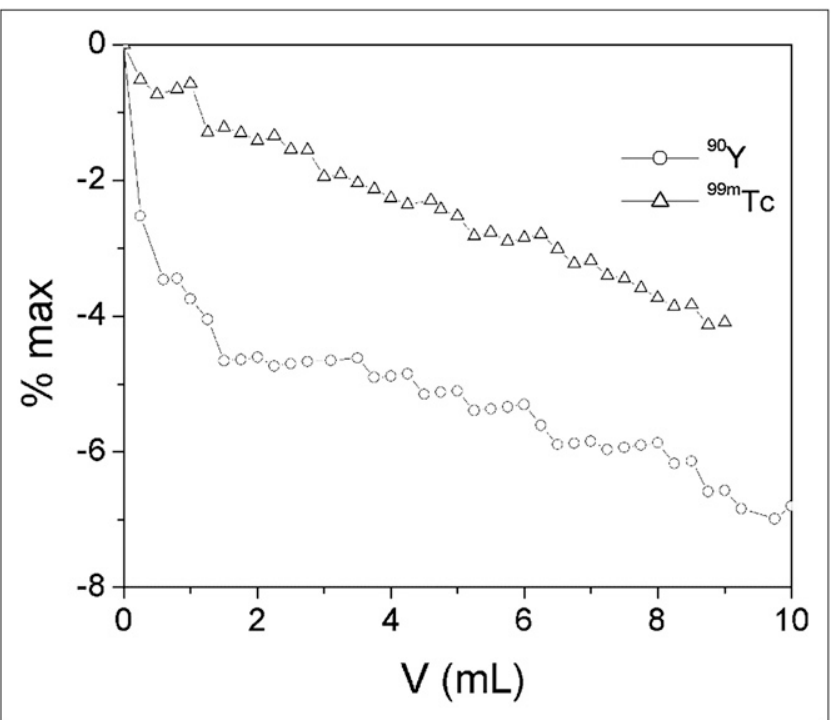

FIGURE 4. Percentage variation of $A_{a p p}$ measured with same activity for both radionuclides ( ${ }^{90} \mathrm{Y}$ and ${ }^{99 \mathrm{mT}} \mathrm{T}$ ) and with volume varied using $10-\mathrm{mL}$ syringe.
SD obtained at each point. On the right axis is shown the percentage deviation to a mean value over $360^{\circ}$ of $A_{\text {app }}$ for a source of about $100 \mathrm{MBq}\left(A_{a p p}=495\right.$ arbitrary units). A total angular variation of almost $4 \%$ over $360^{\circ}$ was found for this particular dose calibrator. For a given calibration factor we can thus write, if the source and holder position were not considered for calibration factor determination, that $\delta A_{2} / A$ is, for this particular dose calibrator, approximately $0.04(4 \%)$.

\section{DISCUSSION}

Even with a careful standard methodology, some oftenunnoticed errors might still be present during calibration factor determination. These will affect both the activity measurement and the dose expected for therapeutic purposes. The geometry of all the devices in the dose calibrator well can originate unexpected sources of error. The characterization of some of these uncertainties and error sources for a particular dose calibrator model (for which the results can be generalized to others) are usually not mentioned in the literature.

Figure 2 shows clear differences between the 3 normalized profiles. We notice a clear difference in the uniformity of the activity response, more visible in the vicinity of the aperture of the well. The well in/out transition change is broader in the ${ }^{99 \mathrm{~m}} \mathrm{Tc}$ case, where the response curve follows a smoother transition as the point source leaves the dose calibrator, when compared with ${ }^{90} \mathrm{Y}$. This can be explained by the shorter propagation length of $\beta^{-}$radiation in air, when compared with the ${ }^{99 \mathrm{~m}} \mathrm{Tc} 140-\mathrm{keV}$ photons. The differences between the curves corresponding to ${ }^{90} \mathrm{Y}$ with and without the plastic insert can also be explained by extra bremsstrahlung $\mathrm{x}$-rays. These are produced by the interaction of the $\beta^{-}$radiation with the plastic insert (besides the ones resulting from the interaction of the $\beta^{-}$with the

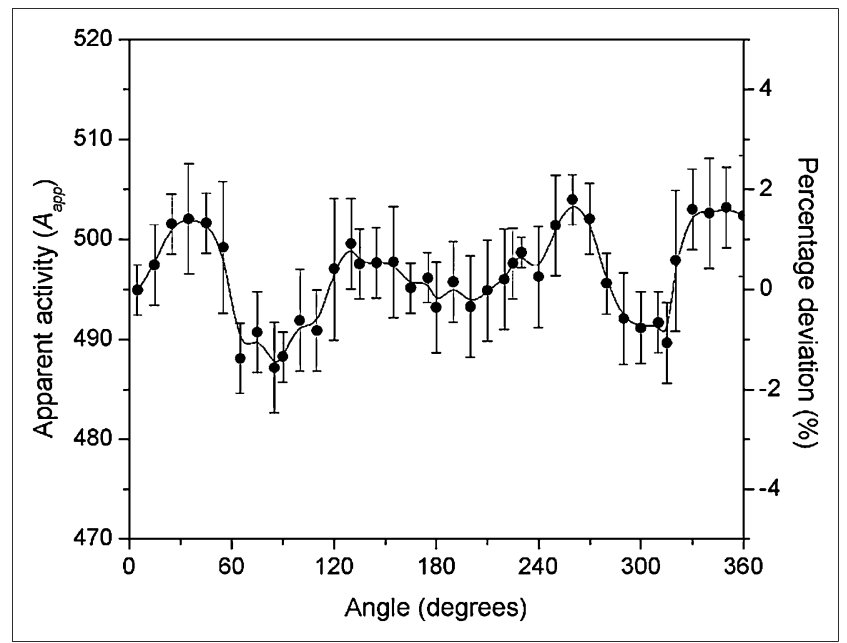

FIGURE 5. Mean values of $A_{a p p}$ as function of source holder angle. Source holder was varied over $360^{\circ}$ by $10^{\circ}$ steps. A $10-\mathrm{mL}$ syringe was used in measurements. 
injected solution, the vial or syringe material, and the metallic inner wall).

\section{Influence of Plastic Source Holder on $A_{a p p}$}

The influence of the plastic source holder on the $A_{a p p}$ as the column of liquid changes inside the container (located at the bottom of the sample holder) can be calculated as follows. $A_{a p p}$ can be written as a function of the depth response of the dose calibrator, $r(x)$, with $x=0$ being the bottom of the metallic well (Fig. 1):

$$
A_{a p p}=k \int_{a}^{b} r(x) d x
$$

where $k$ is an arbitrary constant dependent on the actual activity of the sample and calibration factor used.

A logistic function fits reasonably well the experimental data near the bottom of the plastic sample holder as the responsiveness profile decreases. Thus, the depth responsiveness $r(x)$ can be represented as follows (with $C_{1}, C_{2}, x_{0}$, and $p$ being the fitting parameters):

$$
r(x)=\frac{C_{1}-C_{2}}{1+\left(\frac{x}{x_{0}}\right)^{p}}+C_{2} .
$$

Eq. 3

In the hypothetical case of constant geometric efficiency along the dose calibrator longitudinal axis, $r(x)=1$ for all $x$ and thus,

$$
A_{\text {app }}=k(b-a) \Leftrightarrow k=\frac{A_{a p p}}{b-a} .
$$

In the actual case, the substitution of $r(x)=1$ by the described logistic function will involve a different $A_{a p p}$, $A^{\prime}{ }_{\text {app }}(x)$, which can be calculated as:

$$
A_{a p p}^{\prime}(b-a)=\frac{A_{a p p}}{b-a} \int_{a}^{b}\left(\frac{C_{1}-C_{2}}{1+\left(x / x_{0}\right)^{p}}+C_{2}\right) d x, \quad \text { Eq. } 5
$$

where the integration limits $a$ and $b$ correspond to the bottom and top surface of the cylindric liquid column inside the vial. The calibration factor depends on the vial itself, as is usually assumed in practice, and also on the liquid height inside. For the case under analysis, when $a=6.9 \mathrm{~cm}$ $(6.9 \mathrm{~cm}$ from the bottom of the metallic well bottom surface) and $b$ is changed from $a$ to an arbitrary value $(14 \mathrm{~cm}$ in this case), the percentage error is shown in Figure 6 as a function of $b$ on a $\log$ scale. The variation of the error is also a function of $\Delta x=(b-a)$. The variation of the error as a function of $\Delta x$ is shown on a linear scale in the inset. As an example, if a given $A_{a p p}$ is measured (and the calibration factor is determined) inside the same vial with a column of $3 \mathrm{~cm}$, and if the height of the solution is varied by $5 \mathrm{~mm}$ while the same activity is maintained inside the vial, then an increase of $2.5 \%$ in the $A_{a p p}$ is found. We estimate an error of $\delta A_{3} / A=0.025(2.5 \%)$ as typical for this particular case.

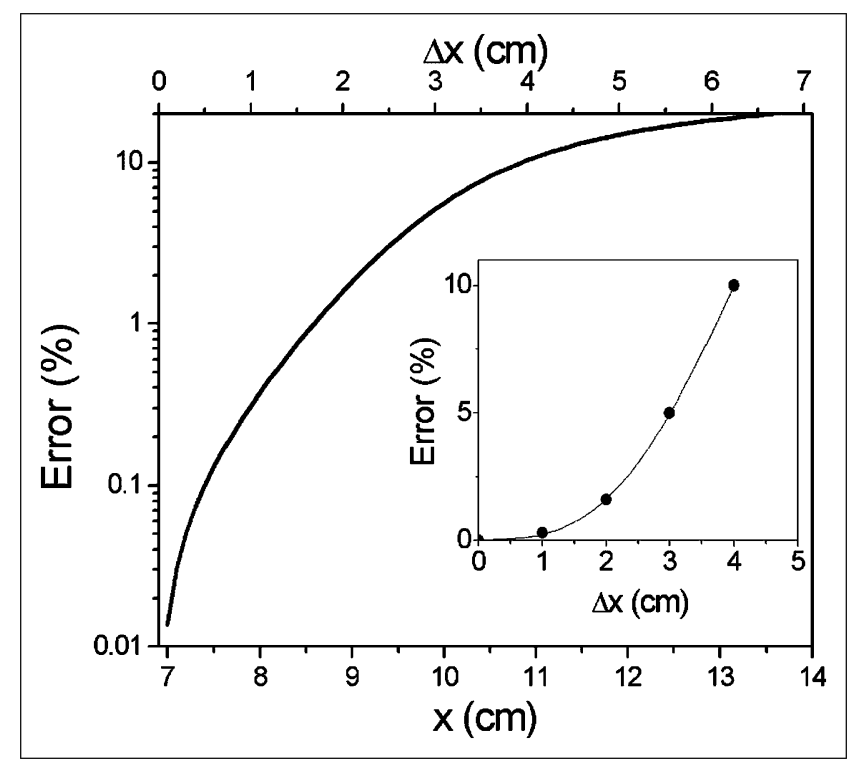

FIGURE 6. $A_{a p p}$ percentage error associated with plastic vial holder (above region iii in Fig. 3) obtained by solving Equation 5. Both logarithmic and linear scale representations are shown.

This estimation of error, as well as the error for $\delta A_{2}$ and $\delta A_{3}$, is sensitive to the volumes used to calculate the calibration factors and for each particular container.

\section{Influence of Liquid Column Length Inside Syringe}

In addition to the sources of error already mentioned, the quantity of the liquid (column length) inside the syringe also influences the $A_{a p p}$. This effect has been found for ${ }^{99 \mathrm{~m}} \mathrm{Tc}$ (15). The same experiment was prepared with ${ }^{90} \mathrm{Y}$, which yielded even larger errors for the latter $\left(4 \%\right.$ for ${ }^{99 \mathrm{~m}} \mathrm{Tc}$ and $7 \%$ for ${ }^{90} \mathrm{Y}$ for a full $10-\mathrm{mL}$ syringe). This difference is mostly due to the initial behavior of the ${ }^{90} \mathrm{Y}$ curve, which is due to the higher absorption of the connecting part of the syringe in the needle connector-much thicker than the syringe material. For a small quantity of liquid, all the activity lies inside this thicker joint, increasing $\beta^{-}$absorption. Once the volume starts to increase, the same activity creates a much larger $A_{a p p}$ because of the lower absorption coefficient of the syringe itself.

\section{Total-Error Propagation Estimation}

After the identification of some of these error sources, we can now calculate the error propagation when measuring the $A_{a p p}$ of a ${ }^{90} \mathrm{Y}$ sample, when the mentioned error sources are present as in the described case.

The first source of error $\left(\delta A_{3} / A=0.025\right)$ comes from the first calibration factor for the reception vial. It depends strongly on the liquid column height inside the vial, as seen in Figure 3. If the liquid height changes, the percentage variation of the activity should be taken into account when any other volume in this vial is measured.

A second source of error $\left(\delta A_{2} / A=0.025\right)$ originates from the angular position of the syringe (or vial) relative to the source holder and to the dose calibration well. 
A third source of error $\left(\delta A_{1} / A=0.02\right)$ originates from the liquid column height inside the syringe, in a way similar to the first error source.

The total error is cumulative and can be calculated according to Equation 1 as:

$$
\frac{\delta A}{A}=\sqrt{0.02^{2}+0.04^{2}+0.025^{2}}=0.051(5.1 \%) .
$$

\section{CONCLUSION}

The activity measurement of the radiopharmaceutical ${ }^{90}$ Y-ibritumomab tiuxetan has great clinical relevance for the treatment of non-Hodgkin lymphoma and has always raised the problem of determining calibration constants for each syringe and vial used during the labeling procedures. In this paper we show that in addition to the existence of container geometry effects (such as volume, shape, and material), there are other sources of error that should be taken into consideration. This may imply that a different strategy must be used to determine calibration factors. A previous individual characterization of the dose calibrator is necessary (through longitudinal profile determination), and other factors, such as the volume inside the container or the rotational position of the source or holder inside the chamber well, can also have a strong influence on the calibration factor outcome and should be considered.

The error can reach values of up to approximately 5.1\% in the CRC-15R using the standard plastic sample holder (Fig. 1) for the conditions stated in the example. This error is as much as the recommended maximum of 5\% uncertainty for therapeutic dose calibration and can increase with less favorable conditions.

Some of these problems can be minimized for each dose calibrator model. The angular response error can be minimized by keeping the holder in the same position relative to the dose calibration well during both calibration factor determination and activity determination. The error due to syringe liquid column height can also be corrected by multiplying the $A_{a p p}$ value by a factor dependent on the volume obtained from the data shown in Figure 4.

If the errors are considered for both the calibration factor determination and the activity measurement, a total error much less than $5 \%$ (excluding possible fluctuations of the dose calibration over short times and intrinsic dose calibrator inaccuracy) can be achieved.

\section{ACKNOWLEDGMENTS}

Comments and suggestions raised by Gregory King and Luís Cunha are highly appreciated. We thank the 3 referees and the associate editor for their critical comments and Gregory King for reviewing the English.

\section{REFERENCES}

1. Selwyn RG, Nickles RJ, Thomadsen BR, DeWerd LA, Micka JA. A new internal pair production branching ratio of ${ }^{90} \mathrm{Y}$ : the development of a non-destructive assay for ${ }^{90} \mathrm{Y}$ and ${ }^{90} \mathrm{Sr}$. Appl Radiat Isot. 2007;65:318-327.

2. Nickles RJ, Roberts AD, Nye JA, Converse AK, Barnhart TE, Rodriguez MA. Assaying and PET imaging of ytrium-90: 1>>34 ppm>0. IEEE Nucl Sci Symp Rec. 2004;6:3412-3414.

3. Lhommel R, Goffette P, Van den Eynde M, et al. Yttrium-90 TOF PET scan demonstrates high-resolution biodistribution after liver SIRT. Eur J Nucl Med Mol Imaging. 2009;36:1696.

4. Calibration and Usage of "Dose Calibrators" Ionization Chambers for the Assay of Radionuclides. Washington, DC: American National Standards Institute; 1986. Document N42:13-1986.

5. Quality Control of Nuclear Medicine Instruments. Vienna, Austria: International Atomic Energy Agency; 1991. TECDOC-602.

6. The 2007 recommendations of the International Commission on Radiological Protection: ICRP publication 103. Ann ICRP. 2007;37(2-4).

7. Medical Electric Equipment: Radionuclide Calibrators-Particular Methods for Describing Performance. Geneva, Switzerland: International Electrotechnical Commission; 1994. Report 61303.

8. A Handbook of Radioactivity Measurements Procedures. 2nd ed. Bethesda, MD: National Council on Radiation Protections and Measurements; 1985. Report 58.

9. Zimmerman BE, Herbst C, Noremberg JP, Woods MJ. International guidance on the establishment of quality assurance programmes for radioactivity measurement in nuclear medicine. Appl Radiat Isot. 2006;64:1142-1146.

10. Parkin A, Sephton JP, Aird EGA, Hannan J, Simpson AE, Woods MJ. Protocol for establishing and maintaining the calibration of medical radionuclide calibrators and their quality control. In: Proceedings of the Joint IPMS/BIR Meeting on Quality Standards in Nuclear Medicine, BIR, London, 1992. York, U.K.: Institute of Physics and Engineering in Medicine. 1992:60-77. Report 65.

11. Schultz MK, Cessna JT, Anderson TL, et al. A performance evaluation of ${ }^{90} \mathrm{Y}$ dose calibrator measurement in nuclear pharmacies and clinics in the United States. Appl Radiat Isot. 2008;66:252-260.

12. Zimmerman BE, Cessna JT, Millican MA. Experimental determination of calibration settings for plastic syringes containing solutions of ${ }^{90} \mathrm{Y}$ using commercial radionuclide calibrators. Appl Radiat Isot. 2004;60:511-517.

13. Stabin MG, Brill AB. State of the art in nuclear medicine dose assessment. Semin Nucl Med. 2008;38:308-320.

14. Taylor JR. An Introduction to Error Analysis. 2nd ed. Sausalito, CA: University Science Books; 1997.

15. Santos JA, Carrasco MF, Lencart J, Bastos AL. Syringe shape and positioning relative to efficiency volume inside dose calibrators and its role in nuclear medicine quality assurance programs. Appl Radiat Isot. 2009;67:1104-1109. 